\title{
Rammya Mathew: Reframing the language around error
}

\author{
Rammya Mathew GP \\ London
}

In medicine, we often equate being wrong with having made a mistake. But we should remember, as the 19th and 20th century physician William Osler observed, that "medicine is a science of uncertainty and an art of probability." So, even when we have made the soundest of decisions, we may still get it wrong.

Our knee jerk response, which has been indoctrinated in us as part of the culture of appraisal, is that we should automatically change the way we practise whenever we make a wrong call. This is certainly appropriate when we've made a mistake. But it may not be when an event with a poor outcome occurs against the odds.

Atypical presentations leading to delayed or missed diagnoses are par for the course, as are rare or unpredictable complications that couldn't have been foreseen.

I was reminded of this when reading of a case in the mainstream media, about a 2 year old girl whose diagnosis of lung cancer was "missed by six doctors." She presented with a persistent cough and reduced appetite since starting nursery at 6 months of age, and her symptoms were attributed each time to an upper respiratory tract infection. Without knowing the specifics of the case, it wouldn't be right to comment, but I'm sure most GPs recognise this as a common history and a very rare diagnosis in a 2 year old.

I wonder how the doctors involved will allow this incident to shape their practice. They may organise more chest $\mathrm{x}$ rays, or perhaps they'll lower their threshold for seeking specialist assessment in an unwell child. This could have consequences for both the healthcare system and its patients: referral rates increase, putting pressure on the system, and more patients endure needless investigations.

The psychologists Daniel Kahneman and Amos Tversky described this phenomenon as "availability": the tendency to overestimate an event's likelihood because of how recent it is in the memory or how unusual or emotionally charged it was. The literature detailing the impact of such cognitive biases in medical decision making is expansive. But proven solutions are few and far between. Reframing the language around medical error would be a good start, so that we clearly distinguish the errors that are genuine and need addressing from the inevitable misfortunes we're bound to encounter as part of a career in medicine. When in doubt we should be able to invite peer or even external review of our decision making_-but assessors must be careful not to allow their judgment of these decisions to be clouded by negative outcomes that couldn't reasonably have been foreseen. And perhaps our appraisers should also be routinely asking us to reflect on the times when we got it wrong but concluded that changing our practice was not going to improve matters for anyone.

Competing interests: I have read and understood BMJ policy on declaration of interests and declare that I have no competing interests.

Provenance and peer review: Commissioned; not externally peer reviewed.

Rare cancer: Kirkcaldy mum feels daughter was let down. BBC News 2019 Mar 1. https: //www.bbc.co.uk/news/uk-scotland-edinburgh-east-fife-47415190.

Published by the BMJ Publishing Group Limited. For permission to use (where not already granted under a licence) please go to http://group.bmj.com/group/rights-licensing/ permissions 